Groupe de lecture critique 



\section{COMPOSITION DU GROUPE DE LECTURE CRITIQUE}

Agence de l'environnement et de la maîtrise de l'énergie (Ademe)

Jean-Marc MÉRILLOT Chef du service Programme recherche

Bureau de recherches géologiques et minières (BRGM)

Christian FOUILLAC Directeur de recherche-Centre scientifique et technique

Commissariat à l'énergie atomique (CEA)

Jean-Claude DUPLESSY Laboratoire des Sciences du climat et de l'environnement (UMR 1572)

Centre de recherche pour l'ingénierie de l'agriculture et de l'environnement (Cemagref)

Philippe DUCHÈNE Chef du département Milieux aquatiques

Centre de coopération internationale en recherche agronomique pour le développement (Cirad)

Jean-Pierre BOUILLET Chef de l'unité de recherche Fonctionnement et pilotage des écosystèmes de plantation

Centre national de la recherche scientifique (CNRS)

André MARIOTII

Directeur scientifique adjoint - Insu/SDU

Institut français du pétrole (IFP)

Jacqueline LECOURTIER Directrice scientifique 
Institut français de recherche pour l'exploitation de la mer (Ifremer)

Jean-Luc DÉVENON Conseiller scientifique du Président

Instifut national de la recherche agronomique (Inra)

Jérôme BALESDENT Chef du département Environnement et agronomie

Institut de recherche pour le développement (IRD)

Roland POSS Directeur de recherche - Unité de recherche Solutions - Montpellier

Institut de radioprotection et de sûreté nationale (IRSN)

Sabine CHARMASSON IRSN- Direction de l'Environnement et de l'intervention (DEI) - Service d'Étude et de surveillance de la radioactivité dans l'environnement (SESURE) - Laboratoire d'Études radioécologiques en milieu continental et marin (LERCM) - La Seyne-sur-Mer

\section{Musée national d'histoire naturelle (MNHN)}

Bernard BODO Département Régulation, développement et diversité moléculaire (UMR 5154)

\section{Office national des forêts (ONF)}

Luc CROISÉ Direction technique - Département Recherche et développement 


\section{Scope France}

Gilles PINAY

Directeur de recherche au CNRS - Département Fonctionnement des écosystèmes au Centre d'écologie fonctionnelle et évolutive de Montpellier

Les membres du Groupe de lecture critique, désignés par le président ou le directeur général de leur établissement, ont examiné le texte du rapport puis, au cours d'une réunion qui s'est tenue à l'Académie des sciences le 10 mai 2006, ont entendu la présentation de M. Georges Pédro, animateur du groupe de travail, et se sont exprimés.

Is ont formulé des remarques, dont certaines ont été intégrées, avec leur accord, dans le rapport; cinq commentaires font l'objet de contributions signées des auteurs : elles sont présentées ci-après. 


\section{COMMENTAIRE DU CENTRE DE RECHERCHE POUR L'INGÉNIERIE DE L'AGRICULTURE ET DE L'ENVIRONNEMENT (CEMAGREF)}

Philippe Duchène

Chef du département Milieux aquatiques

L'approche biogéochimique analysée dans ce rapport vient bien compléter la revue des connaissances (et de leurs lacunes) entreprise dans cette collection de l'Académie des sciences pour permettre de mieux comprendre, donc de mieux décider dans le cadre d'une planète affectée de manière croissante par une population humaine en expansion avec un niveau de vie et de technologies aux effets croissants.

Même si, comme le souligne l'introduction, les contributions présentent une diversité de formes, le tableau ainsi brossé permet de mettre en exergue des priorités de recherche qui nous apparaissent pertinentes.

Parmi celles-ci, il nous apparaît que deux objets d'étude pourront s'avérer déterminants pour fonder une maîtrise de l'ampleur des impacts à très court terme (géologiquement parlant) et mieux comprendre voire prédéterminer les phénomènes de long terme. Nous sommes d'ailleurs heureux de constater la coïncidence avec les objets de la troisième partie de l'ouvrage « secteurs de recherche à développer ".

Il s'agit d'une part de l'activité des micro-organismes, maillon fondamental pour expliciter les flux d'éléments. Cet intérêt devient certain avec les évolutions techniques très rapides, les investigations génomiques de masse, ef l'accès de plus en plus aisé (et d'un coût supportable) par les omiques et la biogéochimie isotopique à l'identification des fonctions métaboliques actives ou potentielles des micro-organismes de l'environnement même non cultivables.

$D^{\prime}$ autre part beaucoup reste à découvrir sur les formes, les rôles des matières organiques d'origine naturelle ou anthropique, la complexation ou la séquestration de métaux ou de toxiques organiques et les cinétiques correspondantes, aussi bien d'ailleurs dans les sols que dans les eaux. 



\section{COMMENTAIRE DU CENTRE DE COOPÉRATION INTERNATIONALE EN RECHERCHE AGRONOMIQUE POUR LE DÉVELOPPEMENT (CIRAD)}

Jean-Pierre Bouillet

Chef de l'unité propre de recherche Fonctionnement et pilotage des écosystèmes de plantation

Le Cirad considère que la compréhension des cycles biogéochimiques est une des clés pour la mise au point de pratiques culturales permettant d'assurer une production soutenue et durable des écosystèmes, à même de satisfaire les besoins des populations du Sud tout en limitant les impacts négatifs sur l'environnement. Le Cirad a identifié ce domaine de recherche comme prioritaire et souligne l'intérêt stratégique que revêt donc à ses yeux ce document.

La structure du rapport, qui traite d'abord du comportement biogéochimique d'un certain nombre d'éléments pivots puis développe une approche par milieu, est particulièrement pertinente et sans doute la seule possible, pour traiter d'un sujet aussi vaste dans un format destiné en premier lieu aux décideurs.

Le Cirad est en accord avec le caractère prioritaire des recherches à mener sur la nature, le rôle et les fonctions de la matière organique - qui joue un rôle clé de la fertilité des sols en milieu tropical - en microbiologie des sols, indispensable pour caractériser la dynamique des principaux éléments minéraux dans le sol et sur la spéciation des éléments chimiques. La nécessité de développer différentes approches de modélisation en biogéochimie est mise en évidence avec justesse, ainsi que l'importance de la prise en compte de différentes échelles spatiotemporelles. Ce dernier point paraît particulièrement important pour passer de l'échelle locale où sont étudiés les processus, aux échelles supérieures, où se déclinent prioritairement les enjeux de développement (on peut ainsi citer l'exemple du passage de la parcelle au bassin versant ou à la région pour la quantification de la production des écosystèmes ou des impacts environnementaux des pratiques culturales). 
D'autres domaines doivent rester prioritaires pour le Cirad comme la dynamique et les conséquences de l'acidification ou de la salinisation des sols sur les cycles biogéochimiques et la production des cultures.

Le Cirad est en plein accord avec le rapport qui met en avant la nécessité de développer une approche systémique et multidisciplinaire pour étudier les cycles biogéochimiques. Mais il lui paraît alors dommage que le document ne souligne pas assez l'intérêt majeur de conduire des recherches communes sur les processus du fonctionnement hydrique, carboné et minéral des écosystèmes, et sur les interactions entre ces processus. Le Cirad regrette ainsi la faible part accordée au rôle de la plante dans les cycles biogéochimiques - à l'exception notable du chapitre sur les écosystèmes forestiers - malgré son rôle essentiel dans le cycle biologique via les transferts sol $\leftrightarrow$ plante des éléments, mais également dans le contrôle des populations de micro-organismes et des propriétés physicochimiques des sols.

Le Cirad soutient les conclusions du rapport sur la nécessité de données spécifiques sur le long terme via l'existence d'observatoires permanents et l'organisation de réseaux d'observation. C'est dans cette logique que le Cirad a mis en place un certain nombre de dispositifs d'étude des cycles biogéochimiques sur les écosystèmes de plantation tropicaux et est intégré à l'ORE F-ORE-T. $\mathrm{Ce}$ pendant la pérennité de ces observatoires et réseaux ne peut être assurée que si des moyens fléchés sont consacrés sur la durée aux institutions en charge de leur conduite.

Enfin le Cirad regrette la trop faible place consacrée à l'utilisation des résultats des recherches pour la mise au point d'outils de gestion durable des écosystèmes (indicateurs...) ainsi qu'aux voies à privilégier et aux moyens à mettre en œuvre pour l'appropriation de ces outils par les acteurs. Plus généralement, il aurait été intéressant que le transfert des connaissances au développement fasse l'objet de recommandations dans le chapitre final du rapport, en particulier en mettant en avant la nécessité d'une meilleure interaction avec les sciences sociales. 


\section{COMMENTAIRES DE L'INSTITUT FRANÇAIS DU PÉTROLE (IFP)}

Jacqueline Lecourtier

Directrice scientifique

Le rapport de l'Académie des sciences concernant les cycles biogéochimiques aborde un sujet majeur quant à la connaissance de la dynamique de notre environnement et de ses implications sociétales.

Comprendre le fonctionnement des cycles des différents éléments dans la nature est une condition sine qua non pour une gestion raisonnée des ressources de notre planète dans une optique de développement durable. De ce point de vue, optimiser l'utilisation des "biens et services écosystémiques ", s'inspirer des processus naturels de régulation des écosystèmes pour restaurer les environnements perturbés, et cela à toutes les échelles d'espace, sont des enjeux cruciaux.

Ce texte fournit des éléments d'information permettant à la fois :

- d'appréhender la notion de cycle biogéochimique et les approches scientifiques mises en œuvre pour les étudier ;

- d'évaluer le niveau de nos connaissances dans ce domaine ;

- de recommander des orientations techniques bien ciblées.

L'organisation de ce document, qui ne prétend pas être exhaustif, est au premier abord un peu déconcertante car il ne répond pas à une logique didactique. Cependant, l'approche choisie est très riche puisqu'elle permet d'une part de faire ressortir les grandes démarches d'étude des cycles (première et deuxième partie) : analyse par éléments (au travers de différents milieux) et analyse par milieux (avec prise en compte de différents éléments), d'autre part de mettre en relief les pistes de progrès, tant en termes d'objets ou de processus (microorganismes, rôle de la matière organique aux interfaces biosphère/géosphère, spéciation des éléments...), qu'en termes de moyens (modélisation, réseaux d'observatoires...). 
Cette approche a également le mérite de souligner le caractère extrêmement complexe de ce champ d'étude en raison la forte interconnectivité de l'ensemble des cycles biogéochimiques à toutes les échelles de temps et d'espace, excluant de fait les simples raisonnement de " causes à effets ". " apparaît clairement à la lecture de document que seul un cadre de pensée "systémique » est à même de fournir une plate-forme de travail pluridisciplinaire où modélisation (conceptuelle et numérique), observation et acquisition de données pertinentes doivent s'associer pour contribuer à une véritable vision intégrée. Cette proche « systémique » permettra notamment une interaction réelle des différents acteurs scientifiques et techniques au travers d'un langage commun, d'une mise en réseaux de données cohérentes entre elles, de mise en œuvre de tests numériques, aussi complets et réalistes que possible, et de propositions de prédictions quant au devenir des écosystèmes en réponse aux changements naturels ainsi qu'aux sollicitations et aux pressions anthropiques.

De plus, ce rapport fait apparaître plusieurs orientations lourdes quant aux stratégies de recherche à mettre en place pour véritablement maîtriser ces cycles biogéochimiques et leurs conséquences sur notre environnement et notre économie :

- développer l'interface entre la chimie, la physicochimie, la géologie ef les sciences du vivant;

- mettre en place (ou réorganiser) des observatoires, tout en se donnant les moyens d'un véritable suivi long terme et d'une mise à disposition large des données accumulées (information et accessibilité) ;

- améliorer l'acquisition de données de terrain tant du point quantitatif que qualitatif que du point de vue de la compatibilité interdisciplinaire;

- rationaliser et optimiser les méthodes d'acquisition de données adaptées à chaque type de problème (par exemple, plans d'expérience);

- développer de nouveaux types de capteurs pour élargir le champ des paramètres mesurables;

- mettre au point des méthodes de gestion des grands volumes de données et constituer des bases de données « intelligentes";

- poursuivre le développement de techniques analytiques à haute performance telles que I'ICP/MS;

- améliorer la modélisation des processus et en particulier, intégrer les progrès récents qui ont été réalisés dans le domaine de la simulation des processus à l'œuvre dans les bassins sédimentaires (systèmes pétroliers, hydrogéologie. .. l, ainsi que dans la description des réservoirs pétroliers et notamment, les changements d'échelle et la gestion des incertitudes vio de nouveaux outils mathématiques et statistiques. 
Le cycle biogéochimique du carbone est un sujet au centre des préoccupations de l'IFP, la maîtrise des concentrations en $\mathrm{CO}_{2}$ dans l'atmosphère constituant, pour le futur, un objectif essentiel à atteindre pour continuer à utiliser les énergies fossiles. Mieux connaitre le fonctionnement de ce cycle, et ses interactions avec les autres cycles biogéochimiques, $s$ inspirer de ces processus pour définir des stratégies de gestion de ce problème majeur pour notre société industrielle est un enjeu primordial. 



\section{COMMENTAIRE DE L'INSTITUT NATIONAL DE LA RECHERCHE AGRONOMIQUE (INRA)}

Jérôme Balesdent

Chef du département Environnement et agronomie

Ce rapport pose utilement dans un même ouvrage les bases du fonctionnement passé et présent des cycles biogéochimiques dans plusieurs de leurs aspects. Les conclusions et recommandations sont appréciées, ainsi que l'affirmation de la pertinence de la réflexion thématique. On tire de ce rapport quelques orientations, parfois complémentaires, pour les priorités de recherche et d'acquisition de connaissances.

Parallèlement au changement climatique et au changement de la chimie atmosphérique, le $\mathrm{XXI}^{e}$ siècle sera inévitablement marqué par un impact sans précédent des activités agricoles et sylvicoles, alimentaires et non alimentaires, sur les cycles de $\mathrm{C}, \mathrm{N}, \mathrm{P}, \mathrm{S}, \mathrm{Si}, \mathrm{Al}, \mathrm{Fe}, \mathrm{Ca}, \mathrm{Na}$, pour ne citer que les principaux, sur la répartition de l'eau, de l'alcalinité, des potentiels d'oxydoréductions, sur les spéciations minérales et organiques des surfaces continentales. Les voies peuvent être très directes, comme la manipulation intentionnelle des flux, jusqu'à très indirectes via les impacts écotoxicologiques par exemple. Les impacts potentiels seront multi-échelles, touchant les sites même de l'usage des terres et de la biosphère, leurs interfaces avec les autres milieux, l'océan et l'atmosphère. Les risques de changements irréversibles ou très lentement réversibles, parfois abrupts, comme la dégradation physique des milieux, le changement de spéciation des éléments majeurs des sols, l'érosion, la salinisation, la perte de biodiversité fonctionnelle doivent recevoir une attention prioritaire. Par l'adaptation et le choix de ses systèmes de culture et de production, l'humanité a le potentiel de réduire, de mitiger ou d'aggraver les conséquences des modifications des cycles, qui touchent in fine les services écosystémiques. Aux côtés de la surveillance des dérives des cycles, de la veille des évolutions biogéochimiques émergentes, de l'élaboration de méthodologies appropriées, la connaissance scientifique et la recherche doivent répondre à des objectifs urgents, plus ou moins évoqués de façon transversale dans différents chapitres du rapport. Parmi ceux-ci on peut compter la prise en compte explicite des systèmes de production et des systèmes de culture dans les représentations de la biosphère continentale, de son fonctionnement biogéochimique et des cycles globaux, la représentation 
fine du fonctionnement et de l'évolution des sols au-delà des cycles du carbone, de l'azote et de l'eau, la mise au point de systèmes innovants de culture et de production durables, la mise au point de nouveaux services environnementaux par l'agriculture, la compréhension du fonctionnement et l'éclairage des acteurs et des porteurs d'enjeux politiques, sociaux et économiques impliqués, la formation et la prévention. Ces objectifs sont actuellement centraux à l'Inra, et sont en plein ceux de plusieurs de ses départements et de plusieurs de ses axes stratégiques. Plusieurs unités propres expérimentales ou de service spécialisées contribuent à la mission nationale d'observation. Les approches, menées en collaboration avec les autres organismes, relèvent typiquement de "la démarche systémique à caractère multidisciplinaire » qu'il est nécessaire de renforcer.

Au plan de l'avancement du front des connaissances, l'évolution conjointe des sciences de la vie, de l'écologie et de la géochimie permet d'envisager des avancées prometteuses à l'interface de ces disciplines sur de nombreux aspects des cycles biogéochimiques. Les progrès de l'analyse moléculaire des communautés microbiennes et de leurs activités, couplées ou non aux traçages isotopiques, permettent enfin d'envisager très concrètement la mise en correspondance des biotransformations géochimiques avec leurs acteurs microbiens. On perçoit également le potentiel des sorties de la biologie moléculaire végétale, pour une meilleure compréhension ou maîtrise des transferts et transformations des éléments minéraux et organiques. Réciproquement, les hétérogénéités spatiales et temporelles, périodiques ou non, du fonctionnement géochimique des milieux apparaissent à toutes les échelles comme un élément fort et sous-estimé de structuration des communautés et de la biodiversité. 


\section{COMMENTAIRE DE SCOPE FRANCE (SCIENTIFIC COMMITTEE ON PROBLEMS OF THE ENVIRONMENT)}

Gilles Pinay

Directeur de recherche au CNRS

Le sujet de ce rapport est tout à fait d'actualité, tant du point de vue des découvertes scientifiques majeures qui ont eu lieu ces dernières années dans ce domaine, que du point de vue de l'application de ces recherches dans les différents domaines de l'environnement. En effet, les récentes avancées dans la compréhension des grands cycles et des couplages entre ces cycles ont apporté des éclairages nouveaux sur les mécanismes de régulation de ces cycles, et par là même, sur la prédiction de leur dysfonctionnement. Les études de ces couplages entre cycles en relation avec le cycle de l'eau permettent maintenant de mieux appréhender les conséquences des changements globaux, qu'ils soient anthropiques ou naturels, sur le recyclage et la disponibilité des éléments dans la biosphère.

Dans ce contexte, l'exercice auquel se sont prêtés les auteurs est donc tout à fait louable et pertinent. Cependant, je dois avouer que la lecture de ce rapport $\mathrm{m}^{\prime}$ a laissé un peu perplexe pour les raisons suivantes :

- le chapitre introductif explique pourquoi la biogéochimie est importante mais ne donne pas clairement les objectifs de ce rapport et ne permet pas de savoir à qui il est adressé et pour quoi faire;

- la première partie du rapport traite des éléments séparément. Ce parti pris peut se défendre mais en l'occurrence, il reste très conventionnel et très général. La plupart des chapitres qui le composent donnent une faible part à la partie biologique et le rôle de l'eau en tant que variable de forçage, catalyseur de réaction ou vecteur de transport n'est que rarement évoqué. Les conséquences sur ces grands cycles des grands enjeux environnementaux, tels que l'augmentation de la concentration en gaz à effet de serre ou les diverses manifestations des changements anthropiques ne sont pratiquement pas considérées; 
- la lecture de la deuxième partie qui présente une approche par milieu est elle aussi très descriptive, ce qui n'est pas une critique en soi. Cependant on aurait pu s'attendre à trouver des raisons pour lesquelles l'étude de ces écosystèmes particuliers est pertinente; en quoi ils permettent de mieux répondre aux grandes questions biogéochimiques du moment et comment on peut utiliser les résultats de ces études de milieux particuliers dans d'autres contextes;

- la troisième partie prospective propose un certain nombre de pistes de recherches dans la continuité de ce qui est déjà réalisé, sans que cellesci soient hiérarchisées. Encore une fois, les grands enjeux actuels, tant théoriques qu'appliqués dans ce domaine ne sont pas clairement évoqués. Cette partie manque du souffle et d'une vision pour l'avenir qui puisse faire de ce rapport une base de réflexion stratégique dans le domaine de la biogéochimie. C'est dommage.

En conclusion, i'avoue que je suis assez déçu par ce rapport; peut-être parce que comme les auteurs, $j^{\prime}$ en attendais trop, faute d'avoir eu une claire définition des objectifs de cette analyse critique. 